\title{
Peer reviewers 2014
}

The Editorial Team would like to thank the individuals listed below who generously gave their time and expertise in acting as peer reviewers for the Journal in 2014.

\begin{tabular}{|c|c|c|c|c|}
\hline Abdala, Nadia & Connor, Mary & Grainger, Corinne & Masters, Tracey & Sathiya Susuman, \\
\hline Afolabi, Ebenezer & Cook, Louise & Griffiths, Malcolm & McDonald, Susan & Appunni \\
\hline Andreadis, Natasha & Cooper, Kevin & Guillebaud, John & McNicholas, Colleen & Schunmann, Catherine \\
\hline Appiah-Agyekum, & Costa, Gilka & Haeri, Sina & Mendoza, Nicolás & Schwartz, Sheree \\
\hline Nana Nimo & Critchley, Hilary & Hamoda, Haitham & Menon, Ken & Schwarz, Eleanor \\
\hline Arcara, Jennet & Crook, David & Heller, Rebecca & Mesher, David & Searle, Stephen \\
\hline Armitage, Catherine & Cuschieri, Kate & Hendry, Maggie & Michie, Lucy & Sewell, Eppy \\
\hline Ba Thike, Katherine & Cwiak, Carrie & Hicks, Stephen & Milewski, Robert & Shapiro, Samuel \\
\hline Bacon, Lesley & Das, Anita & Hinde, Andrew & Miller, Elizabeth & Sharma, Suresh \\
\hline Bahamondes, Luis & de Silva, Shamela & Holman, Ruth & Mittal, Monica & Simpson, Paul \\
\hline Baird, Aisling & DeJong, Jocelyn & Homer, Caroline & Moodley, Dhayendre & Singh, Hari \\
\hline Baldwin, Maureen & Delaney, Ros & Hummelshoj, Lone & Moses, Sharon & Stuart, Beth \\
\hline Barber, Tristan & Dempsey, Deborah & Husemeyer, Roy & Mullin, Nicola & Stuart, Gretchen \\
\hline Barran, Diana & Dickson, Jane & Ingham, Roger & Neves-e-Castro, Manuel & Tamang, Anand \\
\hline Bednarczyk, Robert & Dinger, Juergen & Johnson, Mark & Newton, Victoria & Thach, Chia \\
\hline Berer, Marge & Dodge, Laura & Jones, Sian & O'Brien, Paul & Thompson, Kirsten \\
\hline Bhatia, Ramya & Downing, Jordan & Kahlenborn, Chris & O’Connor, Mairead & Timmerman, Dirk \\
\hline Bignall, Jenine & Dulli, Lisa & Katz, Mira L & Ogbonna, Chinwe & Trewinnard, Karen \\
\hline Black, Kirsten & Eboh, Win & Kavanagh, Jayne & Olsen, Jonathan & Tristan, Sigrid \\
\hline Blume, Alison & Edouard, Lindsay & Keeling, June & Oscarsson, Marie & Tristram, Amanda \\
\hline Bowyer, Harriet & Eliason, Sebastian & Keirse, Marc & Panay, Nick & Trussell, James \\
\hline Bracken, Hillary & Elit, Laurie & Kerns, Jennifer & Patnick, Julietta & Unkels, Regine \\
\hline Bray, Isabelle & Elliman, Alyson & Kibuuka, Hannah & Peipert, Jeffrey & van Teijlingen, Edwin \\
\hline Brin, Genevieve & Espey, Eve & Kilbourne-Brook, & Phiri, Sam & Walker, Susan \\
\hline Brown, Sally & Fargie, Fiona & Maggie & Pike, Malcolm & Waller, Jo \\
\hline Brown, Windy & Farina, Antonio & Kingston, Margaret & Pillai, Mary & Webb, Anne \\
\hline Bryant, Amy & Ferreira, Ana Laura & Kirk, Emma & Potts, Malcolm & Weeks, Andrew \\
\hline Cadman, Louise & Flynn, Ann & Kissling, Frances & Quenby, Siobhan & Weisberg, Edith \\
\hline Caird, Lucy & Forbes, Elizabeth & Kosenko, Kami & Quilliam, Susan & Welbourn, Richard \\
\hline Cameron, Sharon & Foster, Angel & Kulczycki, Andrzej & Ramsay, Philippa & West, Lucy \\
\hline Campbell, Martha & Fraser, Ian & Lee, Ellie & Richards, Christina & Wildt, Ludwig \\
\hline Carr, Susan & Frohwirth, Lori & Lie, Mabel & Riggs, Damien & Wilkins, David \\
\hline Chen, Melissa & Furedi, Ann & Longfield, Kim & Rose, Sally & Willcox, Merlin \\
\hline Chen, Zhong & Gainforth, Heather & Lundgren, Rebecka & Routledge, Jackie & Womack, Justine \\
\hline Clark, Caroline & Gazi, Rukhsana & Lyons, Julianne & Rowlands, Sam & Woodcock, Sean \\
\hline Clarke, Jan & Gerressu, Makeda & Ma, Richard & Sahin-Hodoglugil, & Yam, Eileen \\
\hline Cochrane, Rosemary & Glasier, Anna & Makumbi, Frederick & Nuriye & Zegeye, Desalegn \\
\hline Coffey, Patricia & Graff, Lesley & Mansour, Diana & Salazar, Mariano & Zhang, Peng \\
\hline Connolly, Anne & Graham, Yitka & Marlow, Laura & Sasieni, Peter & \\
\hline
\end{tabular}

